\title{
Space charge resonances in two and three dimensional anisotropic beams
}

\author{
I. Hofmann, ${ }^{*}$ G. Franchetti, and O. Boine-Frankenheim \\ GSI, 64291 Darmstadt, Germany \\ J. Qiang and R. D. Ryne \\ LBL, Berkeley, California 94720 \\ (Received 9 October 2002; published 28 February 2003)
}

\begin{abstract}
The theory and simulation of coherent resonant coupling due to space charge in coasting or bunched anisotropic equilibrium beams is presented. Our work confirms that analytical results on coherent oscillations and instabilities of anisotropic KV (Kapchinskij-Vladimirskij) distributions are a valid tool to interpret the findings from $2 \mathrm{D}$ and $3 \mathrm{D}$ self-consistent particle-in-cell simulations for both $\mathrm{KV}$ and waterbag distributions. With reference to rings we discuss space charge coherent tune shifts up to fourth order and introduce a coherent coupled mode coefficient, which enables us to resolve the issue of KV anomalies by relating them to negative energy modes. The second emphasis of this study is with reference to linacs and a detailed discussion of "stability charts" describing resonant regions where approach to equipartition may occur.
\end{abstract}

DOI: $10.1103 /$ PhysRevSTAB.6.024202

PACS numbers: 41.75.-i, 29.27.Bd, 29.17.+w

\section{INTRODUCTION}

The role of space charge in the context of resonances has found adequate attention only in recent years due to the increasing interest in high-power linear as well as circular accelerators. It is now accepted that space charge is not the only source of the incoherent single-particle tune shift moving particles into machine resonances. Space charge itself can drive resonant mechanisms, which may be enhanced further by the free energy from beam anisotropy, and cause dilution of the phase space density and eventually beam loss. Space charge driven resonance effects in rings have been studied more extensively in recent years within the framework of multiparticle computer simulation, largely with the aim of minimizing beam loss for high beam power (see, for instance, Refs. [1-5]). In this particular context the analytical part of the present study helps to clarify questions concerning the shift of resonance conditions, the "coherent tune shift," up to fourth order. Furthermore, the related coherent mode coefficient allows us to distinguish $\mathrm{KV}$-specific (Kapchinskij-Vladimirskij) instabilities from anisotropy dependent instabilities or resonances, which may apply to a broader class of distribution functions as is shown in the simulation part.

The bulk of this study is dedicated to a comprehensive discussion of the effect of different emittances and/or focusing constants - beam anisotropy - known under the terms of "equipartitioning" in linacs [6]. In Ref. [7] a theoretical foundation in terms of a Vlasov-Poisson eigenmode analysis was presented to respond to the discussion of whether anisotropic space charge dominated beams must approach energy equipartition as "thermodynamics," if applicable to beams, would suggest [8-10].

*Electronic address: I.Hofmann@gsi.de
This analytical work was subsequently applied to 3D simulation studies of nonequipartitioned beams [11], where the fourth order difference space charge resonance was identified as the main mode to push beams towards equipartition, if parameters were such as to excite this resonance; furthermore it was used in a comparison of the fourth order difference resonance (also known as Montague resonance [12]) with analytical theory in 2D simulation [13]. The stability charts resulting from this work have been applied to interpret design simulations for the SNS, the superconducting SPL, as well as the ESS linac designs (see Refs. [14-16]).

A characteristic feature of our work is the presence of a confining potential and related betatron motion in all relevant spatial directions. In this regard our work differs from studies of anisotropy between the transverse direction and the "infinite" longitudinal direction of a coasting beam considered originally in Ref. [17] and more recently in Ref. [18]. The anisotropy instabilities of beams with 1 "unbounded" degree of freedom are more closely related to the "Harris instabilities," the first study of a collisionless coupling in anisotropic fully unbounded neutral plasmas [19]. The work presented here should also help to deepen the understanding that the physics of anisotropic bounded beams is controlled by internal resonances limited to certain stop bands. "Thermodynamic" pictures, instead, may be applicable to beams very close to the space charge limit, where the resonance structure gets lost due to the full overlap of resonance bands, and approach to equipartition becomes a universal feature.

\section{ANALYTICAL CONCEPTS}

The analytical basis is the calculation of coherent frequency shifts and growth rates for leading order eigenmodes from the dispersion relations in Ref. [7], which are 
based on perturbations of the anisotropic KV distribution in the transverse phase space of a coasting beam (using coordinates $x$ and $z$ here, instead of the customary $x$ and $y$, for easier association with the transverse $x$ and longitudinal $z$ in later parts of the paper):

$$
f_{0}\left(z, x, p_{z}, p_{x}\right)=\frac{N T \nu_{x} / \nu_{z}}{2 \pi^{2} m \gamma a^{2}} \delta\left(H_{0 z}+T H_{0 x}-m \gamma \nu_{z}^{2} \frac{a^{2}}{2}\right) \text {. }
$$

Here we assume a constant focusing system with arbitrary focusing ratio and emittance ratio. The equilibrium decoupled Hamiltonians, $H_{0 z}$ and $H_{0 x}$, are constants of the motion; $a$ is the beam radius in $z$ (similarly $b$ in $x$ ), and $\nu_{z}, \nu_{x}$ are the corresponding betatron tunes. The energy anisotropy $T$ is the ratio of oscillation energies in $z$ and $x$ which can be written for harmonic oscillators as

$$
T \equiv \frac{a^{2} \nu_{z}^{2}}{b^{2} \nu_{x}^{2}}=\frac{\epsilon_{z} \nu_{z}}{\epsilon_{x} \nu_{x}}
$$

\section{A. Self-consistent resonant frequencies}

The solution of the linearized Vlasov equation leads to dispersion relations for eigenmodes with space charge potentials expanded in polynomials in $z$ and $x$. Eigenmodes are characterized by the order of the leading term in these polynomials, which also determines the symmetry of the spatial distribution. The analytical work of Ref. [7] is explicitly worked out for second, third, and fourth order modes with a calculation of the eigenfrequencies. These eigenmodes can, in principle, be excited resonantly in a circular accelerator by appropriate external field perturbations as was recently shown for round isotropic beams [2]. The tune notation of circular accelerators is used in this section, but the findings can easily be transferred to linear accelerators by interpreting $\nu_{x}, \nu_{z}$ as phase advances per linac focusing period.

The self-consistent condition of resonance with a field perturbation at harmonic $n$ is given by

$$
\omega=m \nu_{z}+l \nu_{x}+\Delta \omega=n,
$$

where we have introduced the "mode tune" $\omega$ in units of the revolution frequency (analogous to the tunes $\nu_{z}, \nu_{x}$ ). $\Delta \omega$ is the coherent shift away from the single-particle resonance condition caused by the self-consistent beam density oscillations, whereas ignoring $\Delta \omega$ would imply a "frozen-in" charge density distribution. Such a selfconsistent resonance crossing was pointed out analytically by Sacherer [20] and studied numerically - using particle-in-cell simulation-in Ref. [21] for an example of fourth order resonance crossing. In order to quantify the coherent effect we first consider symmetric focusing and symmetric beams and rewrite Eq. (3) by adopting the notation introduced in Ref. [3] for a coherent mode coefficient $C_{m k}$ according to

$$
n=m \nu_{0}-m C_{m k} \Delta \nu=m\left(\nu_{0}-C_{m k} \Delta \nu\right) .
$$

Here $m$ is denoting an azimuthal, $k$ a radial mode number, and $\nu=\nu_{0}-\Delta \nu . k$ reflects the order of the perturbed space charge potential and $m$ the multiple of $\nu_{0}$ for vanishing space charge. The advantage of introducing $C_{m k}$ is that they can be seen as a coefficient of attenuation of the single-particle space charge shift $\Delta \nu$ due to the coherent motion. In some cases there is enhancement, with $C>1$, as will be shown below. For the symmetric breathing mode one has the well-known result $C_{22}=1 / 2$, which reflects the strong density perturbation by the cross sectional breathing. This allows increasing $\Delta \nu$ (hence intensity) by a factor of 2 compared with the corresponding single-particle resonance condition $n=2 \nu$; for the quadrupolar mode the coefficient is only $3 / 4$, hence an allowed intensity increase by the factor $4 / 3$, while the general case for split horizontal and vertical tunes is known to lead to a coefficient $C$ close to the arithmetic means, e.g., $5 / 8$, for both modes.

\section{B. Coherent coupled mode coefficient}

For an adequate discussion of the coherent effect for anisotropic beams and nonsymmetric focusing we propose here to extend the definition of Eq. (4) to the more general case of coupling resonances by introducing

$$
C_{m l k} \equiv \frac{m \nu_{0 z}+l \nu_{0 x}-\omega}{m \Delta \nu_{z}+l \Delta \nu_{x}},
$$

which takes into account the coupling by employing the additional mode number $l$ in both, the expression for the total tune shift, and the coupled incoherent tune shift in the dominator. This definition thus gives a normalized coupled mode coherent frequency shift, which should be used in ring machines to determine the exact position of machine resonances. For $C=1$ this shift vanishes, while the coherent frequency may be shifted past $m \nu_{z}+l \nu_{x}$ for $C>1$ (and $m \Delta \nu_{z}+l \Delta \nu_{x}>0$ ). If $C<1$, the intensity can be increased by the factor $1 / C$ compared with the single-particle resonance condition. In Fig. 1 we show an example of dependence of the mode frequencies ("tunes" in units of the revolution frequency) in second order on the tune depression in the $x$ direction $\left(\nu_{x} / \nu_{0 x}\right)$, where we assume $\epsilon_{z} / \epsilon_{x}=2$. Dashed lines in this and following graphs are the corresponding incoherent expressions $m \Delta \nu_{z}+l \Delta \nu_{x}$. In this and following examples the absolute value of tunes is insignificant as we are not studying external resonances driven by the lattice. What matters here is the relative tune depression by space charge.

Note that in the zero space charge limit the mode frequencies coincide with $2 \nu_{0 z}, 2 \nu_{0 x}$ for the envelope breathing and quadrupolar ("even") modes; furthermore $\nu_{0 z} \pm \nu_{0 x}$ for the skewed modes ("odd" or "tilting" mode since the real space ellipse is tilting), which could be excited by skew quadrupoles (linear coupling), or emerge from initial noise by exponential instability of a 


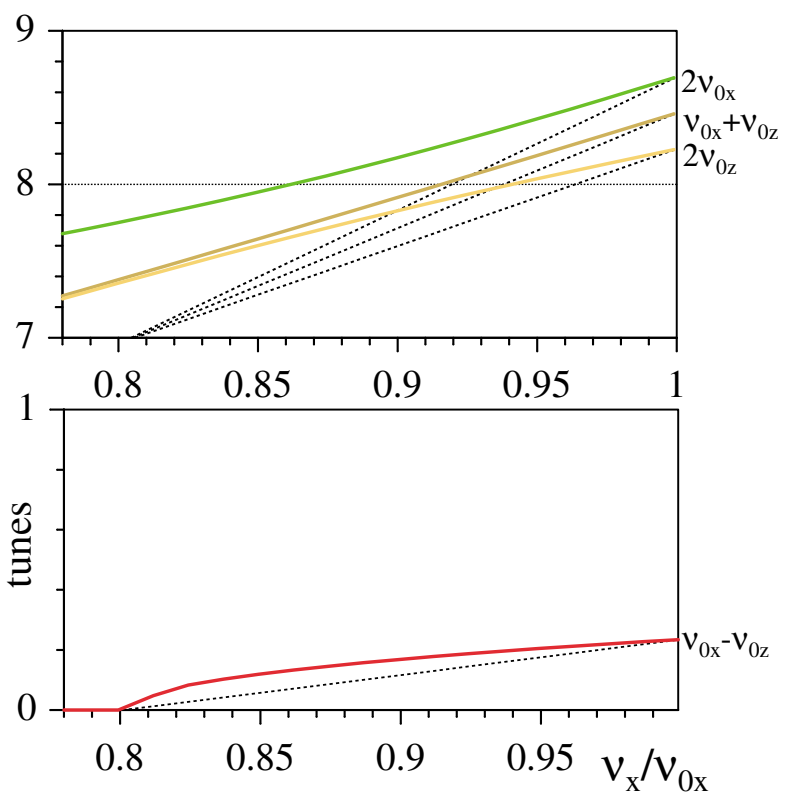

FIG. 1. (Color) Analytic results for second order coherent and incoherent (dashed lines) frequencies with $\epsilon_{z} / \epsilon_{x}=2$ and machine tunes $\nu_{0 z}=4.115, \nu_{0 x}=4.35$.

$z x$ term in the self-consistent space charge potential as will be discussed below. At finite intensities the envelope mode frequencies are shifted upwards as expected, with $C \approx 5 / 8$, if compared with the single-particle expression $m \nu_{z}+l \nu_{x}$. This illustrates the approximately $60 \%$ larger tolerable intensity than would be inferred from Eq. (3) if $\Delta \omega$ were ignored. This effect was also confirmed in the simulations in Refs. [1,3] and applied in a detailed study to the SNS ring in Ref. [4].

For the second order difference skew mode we find $C_{-112} \approx 1 / 2$ for small space charge, whereas for $\nu_{x} / \nu_{0 x}=0.8$ both the coherent frequency and frequency shift vanish in our example, hence $C_{-112}=1$. Exceeding this threshold this "nonoscillatory mode" $(\Re \omega=0)$ adopts a finite imaginary part indicating a pair of solutions, one exponentially unstable and one damped. Such a spontaneous skew mode instability occurs whenever the conditions $\nu_{z} / \nu_{x}>1$ and $\nu_{0 z} / \nu_{0 x}<1$ are simultaneously satisfied [7]. This particular case of second order unstable mode, with no external driving term, plays a role for our coherent interpretation of the Montague resonance phenomenon, where it enhances the effect of the then dominant resonant instability in fourth order.

Proceeding to fourth order modes (Fig. 2) the even mode frequencies merge to the values $4 \nu_{0 z}, 2 \nu_{0 z} \pm 2 \nu_{0 x}$, and $4 \nu_{0 x}$ as well as to $2 \nu_{0 z}$ and $2 \nu_{0 x}$ in the zero intensity limit. These are also the frequencies at which a beam resonates if exposed to a normal octupole [22]; we also obtain odd mode frequencies merging into $3 \nu_{0 z} \pm 2 \nu_{0 x}$, $3 \nu_{0 x} \pm 2 \nu_{0 z}, \nu_{0 z}$, and $\nu_{0 x}$ (not shown here), comparable with those excited by skewed octupoles [22].
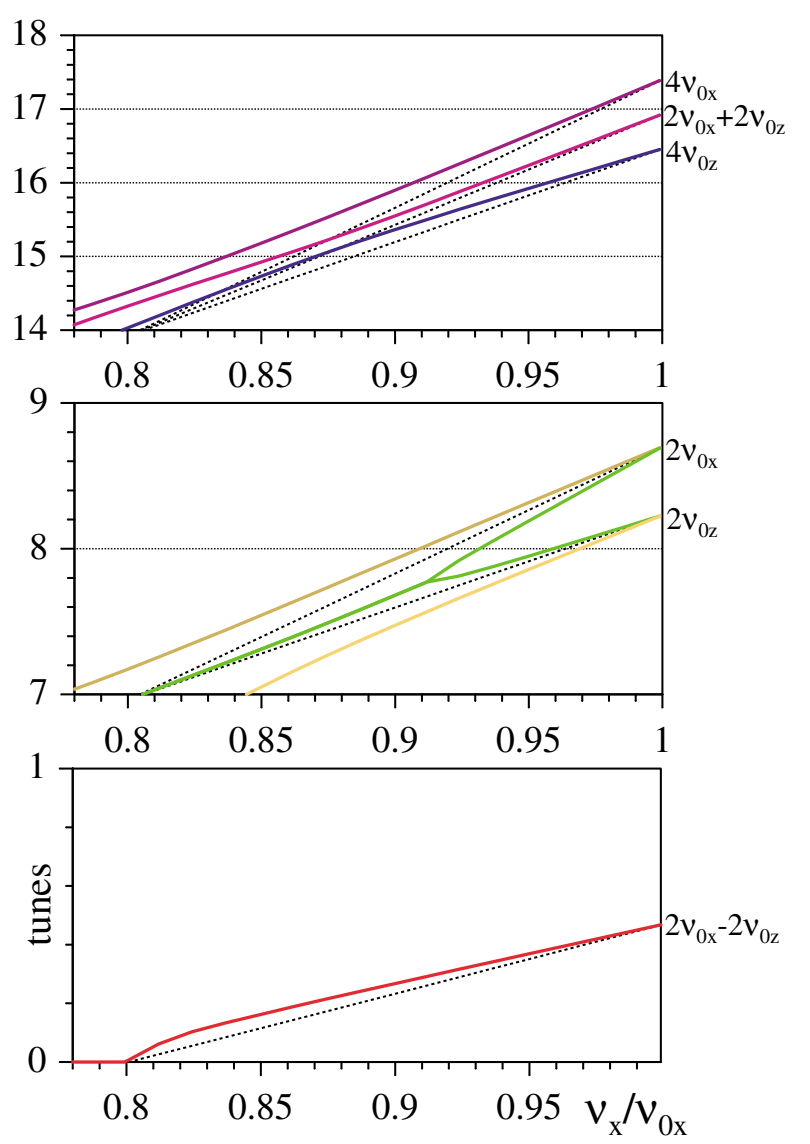

FIG. 2. (Color) Analytic results for fourth order even mode frequencies with $\epsilon_{z} / \epsilon_{x}=2$ and machine tunes $\nu_{0 z}=$ $4.115, \nu_{0 x}=4.35$.

It is noted that for higher order the coherent shifts are reduced as was shown already in the example of Ref. [21]. For the highest frequency branches of the even modes, and using parameters of Fig. 2, we obtain $C_{044}=0.88$, compared with $C_{303}=0.77$ in third order, and $C_{022}=$ 0.65 in second order. Note that these frequencies depend on emittance and tune ratios, but the dependence is relatively weak and the trend seen in this example is a general one.

\section{Negative energy KV modes}

The lower branch in Fig. 2 originating from $2 \nu_{0 x}$ has the peculiar feature of a negative coherent shift, past the single-particle resonance condition $\left(C_{024}>1\right)$; at some intensity it merges with the upper branch originating from $2 \nu_{0 z}$-with positive coherent shift $\left(C_{204}<1\right)-$ into a pair of exponentially growing and damped solutions. Such KV instabilities have been calculated first by Gluckstern [23] for axially symmetric equilibrium beams. For this case it was shown in Ref. [24] that the $\mathrm{KV}$ instabilities should be understood as resulting from a confluence of "negative energy" oscillations with "positive energy" oscillations. Note that growth or damping under conservation of the total energy is only possible for 
energetically "neutral" modes. This distinction between negative or positive mode energy implies that energy must be taken away, respectively, added to the unperturbed beam in order to excite the mode. According to Ref. [25] the existence of modes with negative energy is a peculiarity of the KV distribution as a delta function distribution of the Hamiltonian. Such modes disappear for monotonically decreasing distribution functions, including the waterbag (WB) with its constant distribution function as a marginal case, for which the equilibrium is at a state of minimum energy, hence energy needs to be added to the unperturbed beam to excite a mode [25]. Consistently, monotonically decreasing distributions for isotropic beams are found to be stable.

In Ref. [24] it was shown for symmetric beams that positive or negative energy relates to $\omega / \nu$ increasing, respectively, decreasing with intensity, which is equivalent to $C<1$, respectively, $C>1$. We postulate, and find the 2D simulations fully supporting this, that this classification can be generalized to anisotropic beams. Hence, $\mathrm{KV}$ modes with $C<1$ are positive energy modes for which a corresponding mode is also found for WB beams; but this does not apply to KV modes, and associated instabilities, with $C>1$. For example, the lowest branch originating from $2 \nu_{0 x}-2 \nu_{0 z}$ has $C_{-224}<1$, which shrinks to zero at $\nu_{x} / \nu_{0 x}=0.8$, where transition to instability occurs, similar to the above finding for the second order difference skew mode. To complete the picture we show in Fig. 3 the case of a similar instability of a third order difference even mode, which requires stronger tune splitting to be observed. The lowest frequency branch with $C_{-123}$ approaching unity from below, hence again a positive energy mode, becomes unstable for $\nu_{x} / \nu_{0 x}<0.5$. The negative energy mode originating from $\nu_{0 z}$ is stable in this example. Note that symmetric KV beams are fully stable in third order.

We emphasize that the energy anisotropy in conjunction with the kinetic Vlasov treatment leads to a substan-

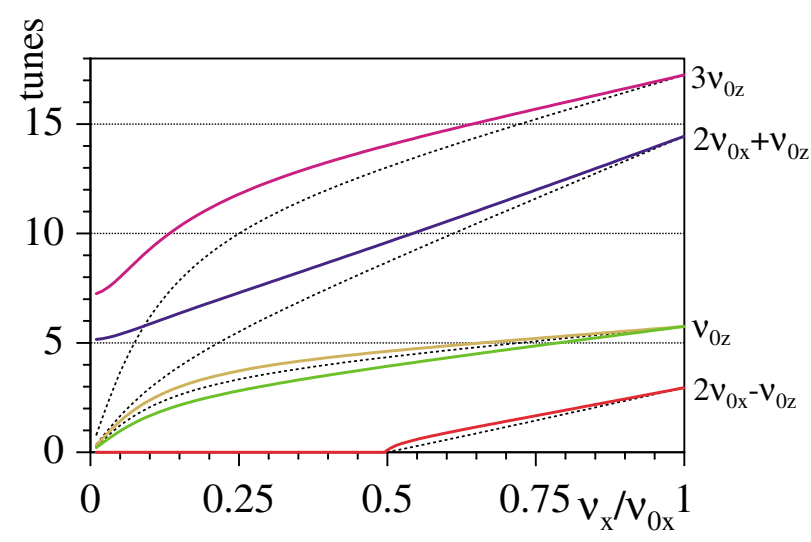

FIG. 3. (Color) Analytic results for third order even mode frequencies with $\epsilon_{z} / \epsilon_{x}=2$ and machine tunes $\nu_{0 z}=$ 5.75, $\nu_{0 x}=4.35$. tial increase in the number of eigenmodes for given order compared with the isotropic KV theory of Ref. [23]. Note that fluid models derived for fully symmetric beams [26] only give the highest frequency branches, but not the lower frequency positive energy modes, which are of kinetic origin (like a fourth order mode branch originating from $2 \nu_{0}$ ). To summarize this section we suggest an unambiguous classification:

(1) Modes with $C>1$ (negative energy modes) are KVspecific modes and should be discarded, likewise the instabilities resulting from a confluence of negative and positive energy modes, which includes all cases of oscillatory instability $(\Re \omega \neq 0)$.

(2) The bulk of the modes has $C<1$ (positive energy modes); hence these modes should be considered also for realistic distribution functions, although the possibility of Landau damping by a sufficiently large tune spread cannot be excluded for some of these modes. There are possibilities of transition to resonant instability at $C=1$, which are driven by the energy anisotropy and which are always found to occur in a nonoscillatory way $(\Re \omega=0)$.

\section{Resonant collective instability}

A more systematic study helps to explore the parameter space where resonant exchange may occur and to verify the positive-negative energy mode concept via the appearance of resonant instability by the subsequent simulation studies. For this purpose it is convenient to create a more comprehensive map of possible unstable solutions of the dispersion relations by plotting them in terms of the betatron tune ratio $\nu_{z} / \nu_{x}$ (which is in general different from the zero space charge tune ratio $\nu_{0 z} / \nu_{0 x}$ ) for a fixed emittance ratio $\epsilon_{z} / \epsilon_{x}$ and thus sample a resonant stop band under constant space charge tune depression in $x$. We first show results for a typical example with modest space charge tune depression (as might occur in a high-current ring) in Fig. 4, using $\epsilon_{z} / \epsilon_{x}=2$ and $\nu_{x} / \nu_{0 x}=0.8$. Cases

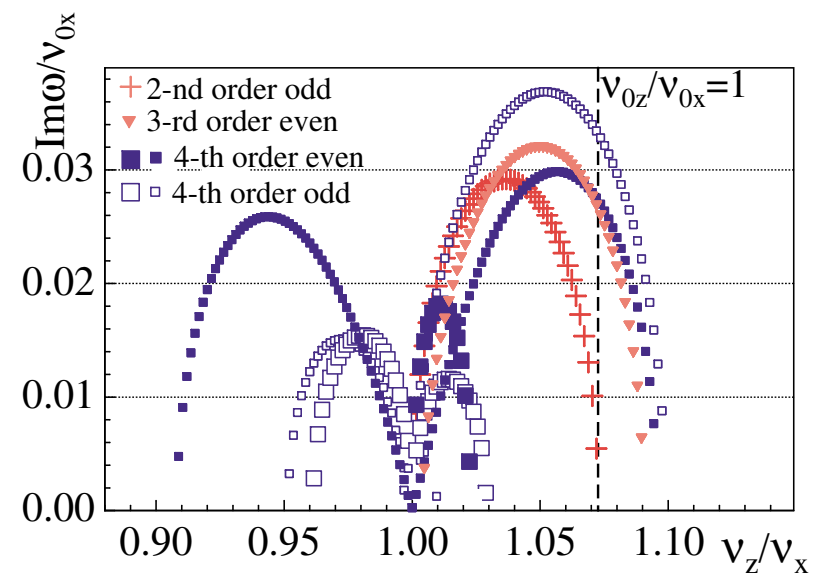

FIG. 4. (Color) Analytic instability growth rates (normalized) for constant $\nu_{x} / \nu_{0 x}=0.8$ and $\epsilon_{z} / \epsilon_{x}=2$ as a function of the betatron tune ratio and including all modes up to fourth order. 
of unstable behavior are marked for later comparison with the simulation: small markers relate to oscillatory $\mathrm{KV}$ instabilities $(\Re \omega \neq 0)$, big markers to nonoscillatory modes $(\Re \omega=0)$. For $\nu_{z} / \nu_{x}=1$ all rates vanish.

Growth rates are in units of $\nu_{0 x}$, hence the typical growth rate of 0.03 in Fig. 4 corresponds to $(2 \pi 0.03)^{-1} \approx$ 5.3 zero space charge betatron wavelengths in the $x$ direction. The dominant modes appear to be of fourth order, which suggests the resonance condition $2 \nu_{z}-$ $2 \nu_{x}+\Delta \omega=0$. Note that the full width of the stop band $0.91 \lesssim \nu_{z} / \nu_{x} \lesssim 1.1$ is controlled by the oscillatory KV instabilities, which are more spread out than the nonoscillatory instabilities. For emittance ratios exceeding five this is reversed, while for equal emittances this band of nonoscillatory modes shrinks to zero, which reflects that energy equipartition is established in this case; only oscillatory "unphysical" KV instabilities are left to induce emittance exchange, surprisingly away from equipartition (as studied recently in Ref. [27].

This particular resonance was the subject of the singleparticle approach-ignoring the coherent $\Delta \omega$-by Montague who considered the fourth order space charge coupling term in an initial Gaussian density distribution, from which it could be concluded that equal tunes should not be taken in a synchrotron with unequal emittances to avoid exchange [12]. Our self-consistent approach assumes no space charge coupling terms in the equilibrium, but shows that fourth order modes may grow, from initial noise, in an unstable way and thus provide coupling. Furthermore it shows the appearance of unstable third order modes, although oscillatory $\mathrm{KV}$ modes, for $\nu_{z} / \nu_{x} \gtrsim 1$, which in a single-particle model would only be expected to appear near $2 \nu_{z}-\nu_{x} \approx 0$, or $\nu_{z}-$ $2 \nu_{x} \approx 0$. In addition, Fig. 4 indicates also instability of the second order difference skew mode.

Analytical growth rates for stronger tune depression pertinent to high-current linacs (where we relate $x$ to the transverse coordinates and $z$ to the longitudinal) show a

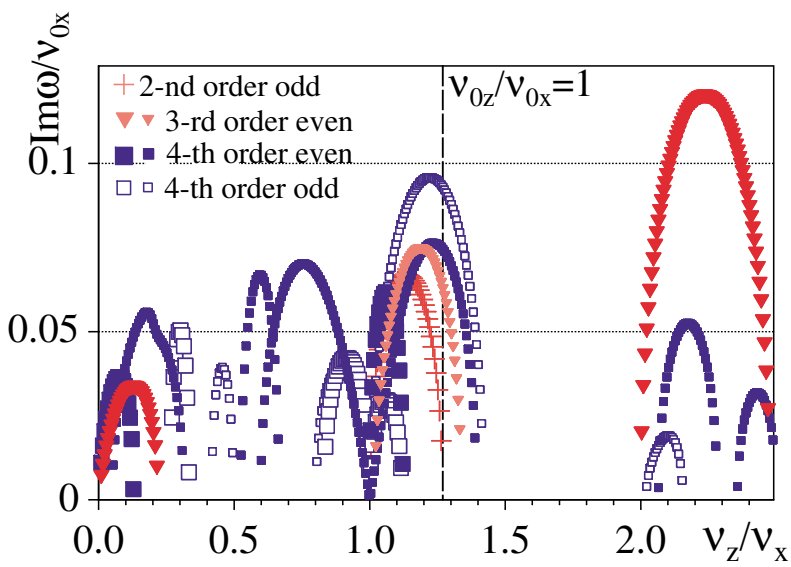

FIG. 5. (Color) Analytic instability growth rates (normalized) for constant $\nu_{x} / \nu_{0 x}=0.5$ and $\epsilon_{z} / \epsilon_{x}=2$. quite similar distribution of modes as in Fig. 4 as long as $\nu_{x} / \nu_{0 x} \gtrsim 0.5$, besides overall increasing values for the rates and broadening of the stop-band width (see Fig. 5).

The appearance and strength of stop bands of resonant instability in the vicinity of integer tune ratios depends on the actual emittance ratio and the amount of energy anisotropy calculated from Eq. (2). The stop-band structure is more clearly identifiable if we discard in the plots the oscillatory KV instabilities, which would actually fill up most of the region left from $\nu_{z} / \nu_{x}=1$. An overview on all of the nonoscillating unstable modes and the variation of stop bands is shown in Fig. 6 for three different emittance ratios.
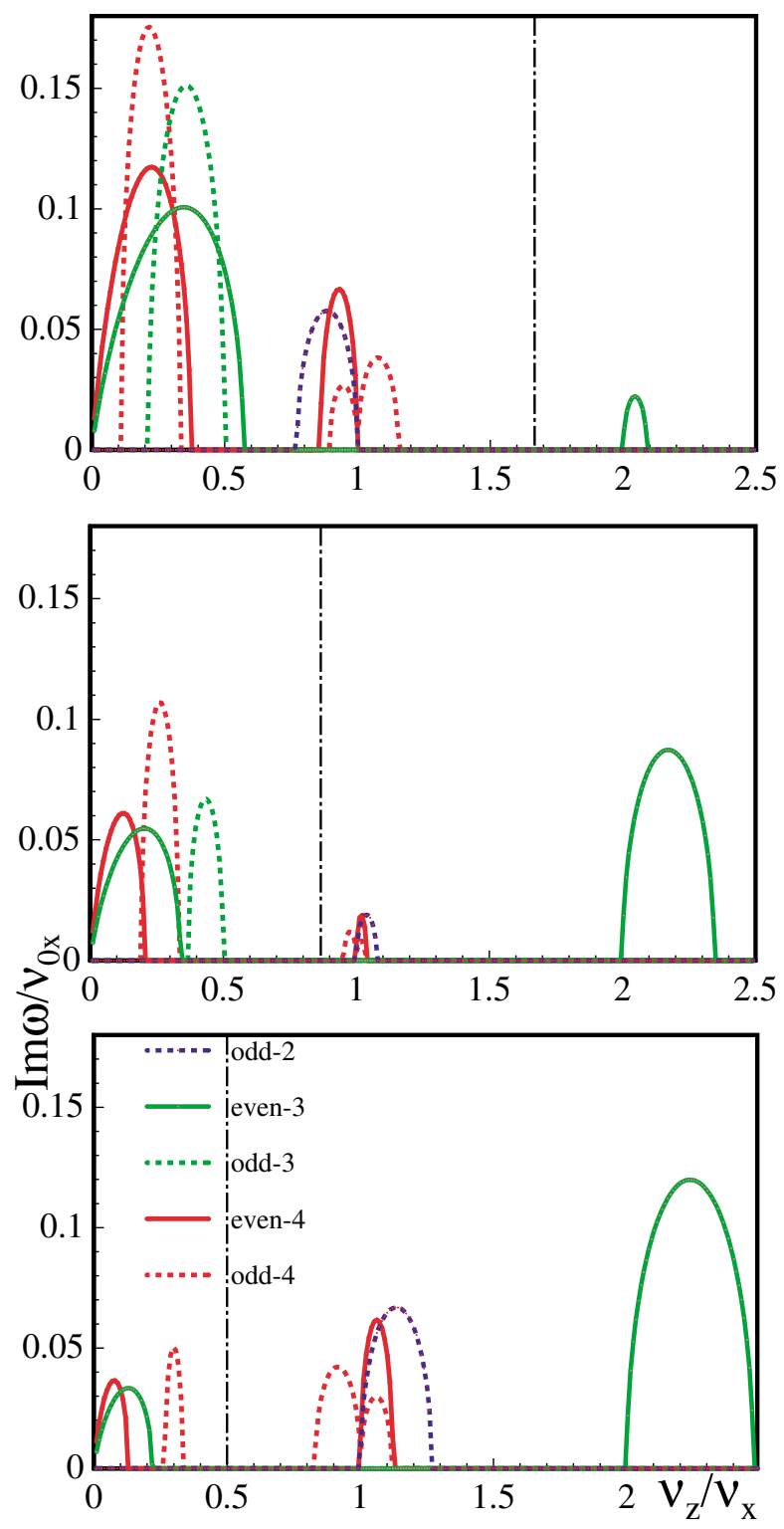

FIG. 6. (Color) Growth rates (normalized) for constant $\nu_{x} / \nu_{0 x}=0.5$ and $\epsilon_{z} / \epsilon_{x}=0.6,1.2$, and 2 (from top to bottom) including only nonoscillating modes up to fourth order. 
The widths of stop bands and the associated growth rates are strongly suppressed at tune ratios where the condition of energy equipartition is satisfied. As an example, the stop band at $\nu_{z} / \nu_{x} \approx 1$ is tiny for the emittance ratio 1.2 and vanishes completely for equal emittances.

\section{2D SIMULATION}

For the study of these resonances beyond the linearized theory, and to clarify the distinction between KV and WB distributions along the lines of negative and positive energy modes, we have employed a standard 2D particle-in-cell code with $5 \times 10^{4}$ simulation particles in a rectangular conducting boundary sufficiently far away from the beam using the MICROMAP code [28]. We have carried out simulations with initial $\mathrm{KV}$ as well as rms matched WB distributions for the same parameter range as in Fig. 4. The WB initial distributions, contrary to KV beams, cannot be generated in a self-consistent way in the case of anisotropy. We fill a 4D hyperellipsoid uniformly and carry out rms matching, which leaves an inevitable initial density mismatch containing fourth, sixth, and higher order modes. This density mismatch is, however, small for the tune depressions considered here. Note that all quantities such as tunes or emittances are understood as rms values.

In Fig. 7 we show a typical example of the dynamical evolution of rms emittances within the resonance stop band at $\nu_{z} / \nu_{x}=1.042$. It is noted that for the KV beam the exchange process shows an exponential rise (from noise nonlinearities) with saturation, but full equipartitioning is not achieved. For the WB, instead, we find immediate exchange. This is not surprising, since the expected main driving force for this resonance is a fourth order term in the space charge potential, which is already of significant size in the initial WB configuration. The saturated emittances are closer to equipartition.

The picture of a dominant fourth order mode in the early stage of the WB case in Fig. 7 is confirmed by the computer animation of the simulation scatter plots in real and phase space as shown in Video 1. The square shape (fourfold symmetry) adopted by the real space distribution near cell 50 (period 15) indicates the dominance of the fourth order deformation and the coherent response. At this time the emittances have also become practically equal. At twice the time there is an indication of a higher order multipole (eightfold symmetry), which is not unusual for collective nonlinear motion.

Results for the rms emittance exchange in each plane-taken after 110 betatron periods in $x$ defined without space charge - are given in Fig. 8. For the KV case the full stop-band width is in excellent agreement with the analytical one of Fig. 4 and reflects the existence of the oscillatory instabilities determining the left and right edges of the stop band. The theoretically found
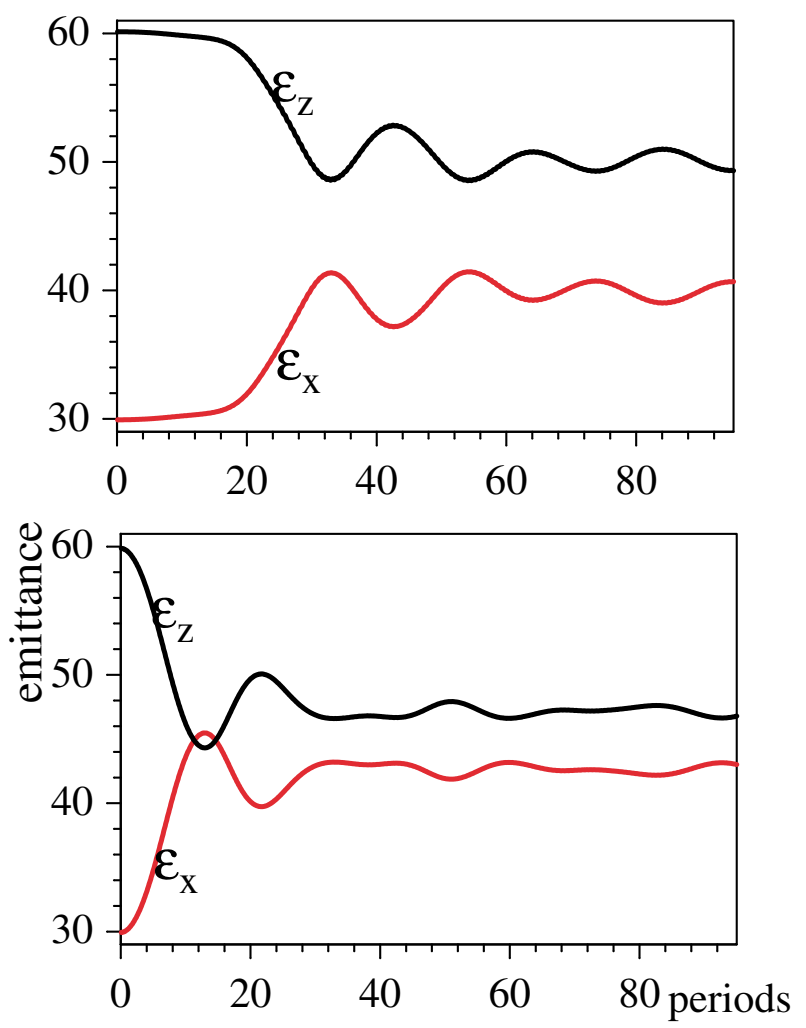

FIG. 7. (Color) Evolution of rms emittances (arbitrary units) for $\nu_{z} / \nu_{x}=1.04, \nu_{x} / \nu_{0 x}=0.8$, and $\epsilon_{z} / \epsilon_{x}=2$ with initial $\mathrm{KV}$ (top panel) and WB (bottom panel). Units on the abscissa are betatron periods in $x$ in the absence of space charge.

absence of instability for $\nu_{z} / \nu_{x}=1$ is also confirmed by the simulation. Left from this point the emittance transfer is, perhaps surprisingly, into the direction of the originally larger emittance $(z)$. The maximum exchange is reached at $\nu_{z} / \nu_{x} \approx 1.073$, where $\nu_{0 z} / \nu_{0 x}=1$, and the second order skew mode ceases to be unstable according to Fig. 4. For the WB the emittance transfer is limited to a tighter stop band, which is consistent with Fig. 4 if the oscillatory KV instabilities (small markers) are discarded, hence $0.96 \lesssim \nu_{z} / \nu_{x} \lesssim 1.073$. In the small band $1.06 \lesssim \nu_{z} / \nu_{x} \lesssim 1.073$ the WB also shows the appearance of the skewing mode instability predicted in $\mathrm{Sec}$. II C as a non-KV mode: emittances are periodically exchanged between $z$ and $x$, similar to a second order difference resonance driven by skew quadrupoles, but here spontaneously driven by space charge. In this band the markers of Fig. 8 are placed to indicate the maximum of the exchange for the WB, which includes overshoot beyond equipartition.

For stronger space charge one finds very similar response bands, though broadened correspondingly. In Fig. 9 we summarize results, taken after 95 betatron periods in $x$ defined without space charge, for WB beams with $\nu_{x} / \nu_{0 x}=0.5$ and different emittance ratios as in Fig. 6.

Comparison with Fig. 6 confirms the observation made above that non-negligible emittance transfer for WB 


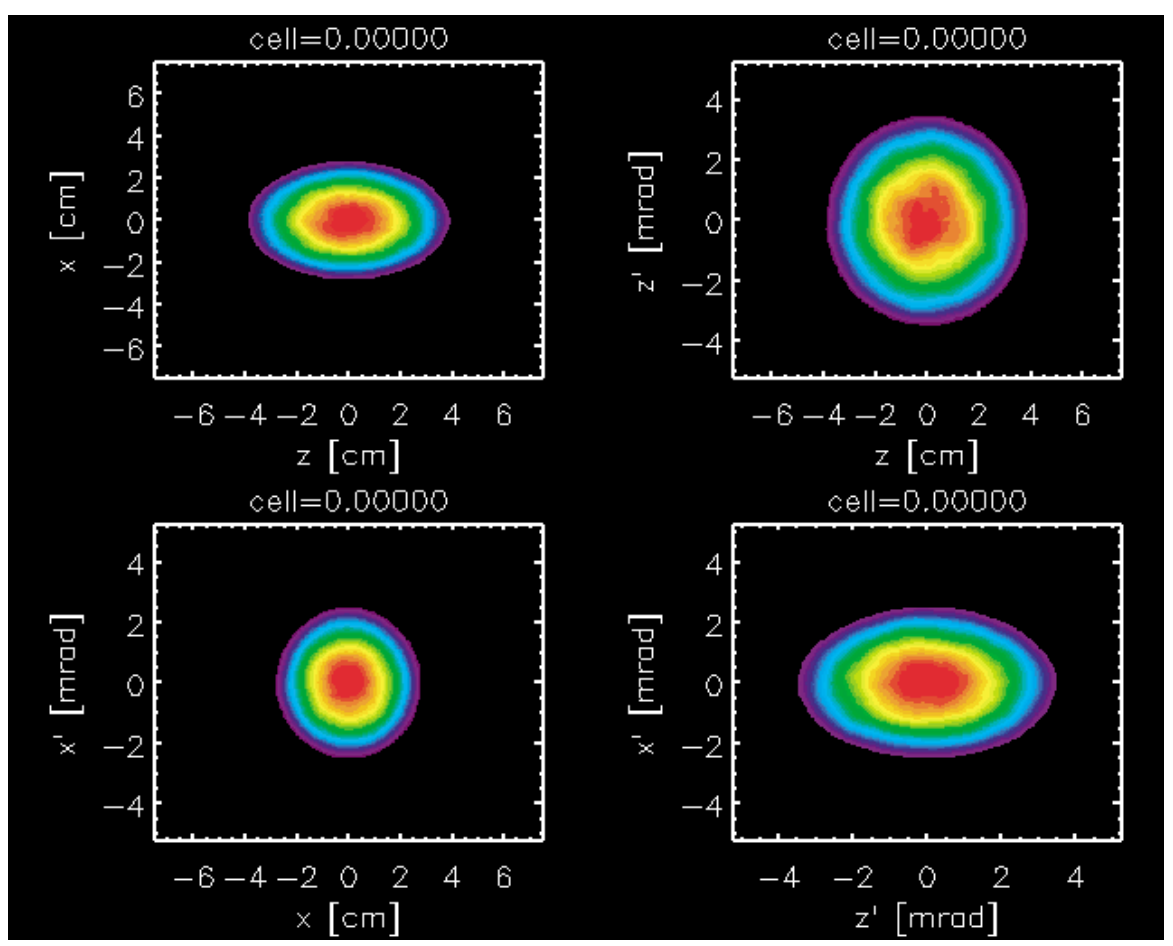

VIDEO 1.Animated results from computer simulation of the WB case of Fig. 7 showing the emittance exchange evolution over 30 periods. Shown are scatter plots in $(z, x),\left(z, z^{\prime}\right)$, and $\left(x, x^{\prime}\right)$ evolving in time, where 1 cell corresponds to 0.3 periods of Fig. 7 . Red indicates maximum point density, and black zero point density.
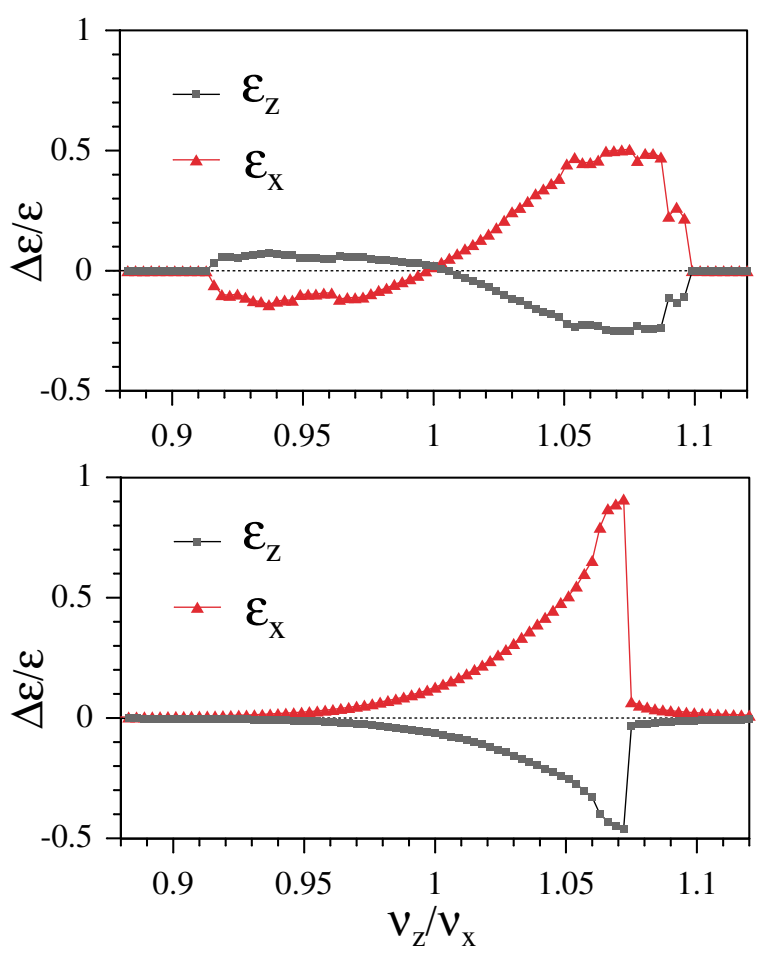

FIG. 8. (Color) Simulation results showing final relative emittance change for initial KV (top panel) and WB (bottom panel) distributions $\left(\nu_{x} / \nu_{0 x}=0.8, \epsilon_{z} / \epsilon_{x}=2\right)$ as functions of the tune ratio. beams is limited to the nonoscillatory modes, while the oscillatory instabilities should be discarded as KV artifact. A further important finding is the absence of additional stop bands for higher than fourth order modes in the simulation. In particular, Fig. 9 shows that there is no evidence of emittance transfer for the WB simulations near tune ratios $\nu_{z} / \nu_{x} \approx 2 / 3,3 / 2$, where fifth order stop bands might be expected. The KV simulation, instead, has indicated a weak effect by these higher order resonances over the considered duration of the runs.

In order to appreciate the role of collective motion we have examined the bottom case of Fig. 9 in a simulation where the initial space charge potential of the WB beam was "frozen in" (Fig. 10). A fourth order resonance stop band at $\nu_{z} / \nu_{x} \approx 1$ is clearly induced by the stationary WB space charge potential, but the maximum emittance exchange is weakened due to the lack of self-consistency; likewise the strong asymmetry of the response curve is lost. For the small tune ratio the focusing in $z$ drops and remains effectively below the static defocusing of the frozen-in WB space charge density, which explains the unbounded emittance growth for $\nu_{z} / \nu_{x} \rightarrow 0$ as artifact. The strong third order resonant instability at $\nu_{z} / \nu_{x} \approx 2$, instead, has no counterpart in the frozen-in simulation due to the absence of sextupolar terms in the stationary WB space charge potential; hence this mode is entirely coherent and requires self-consistent modeling. 


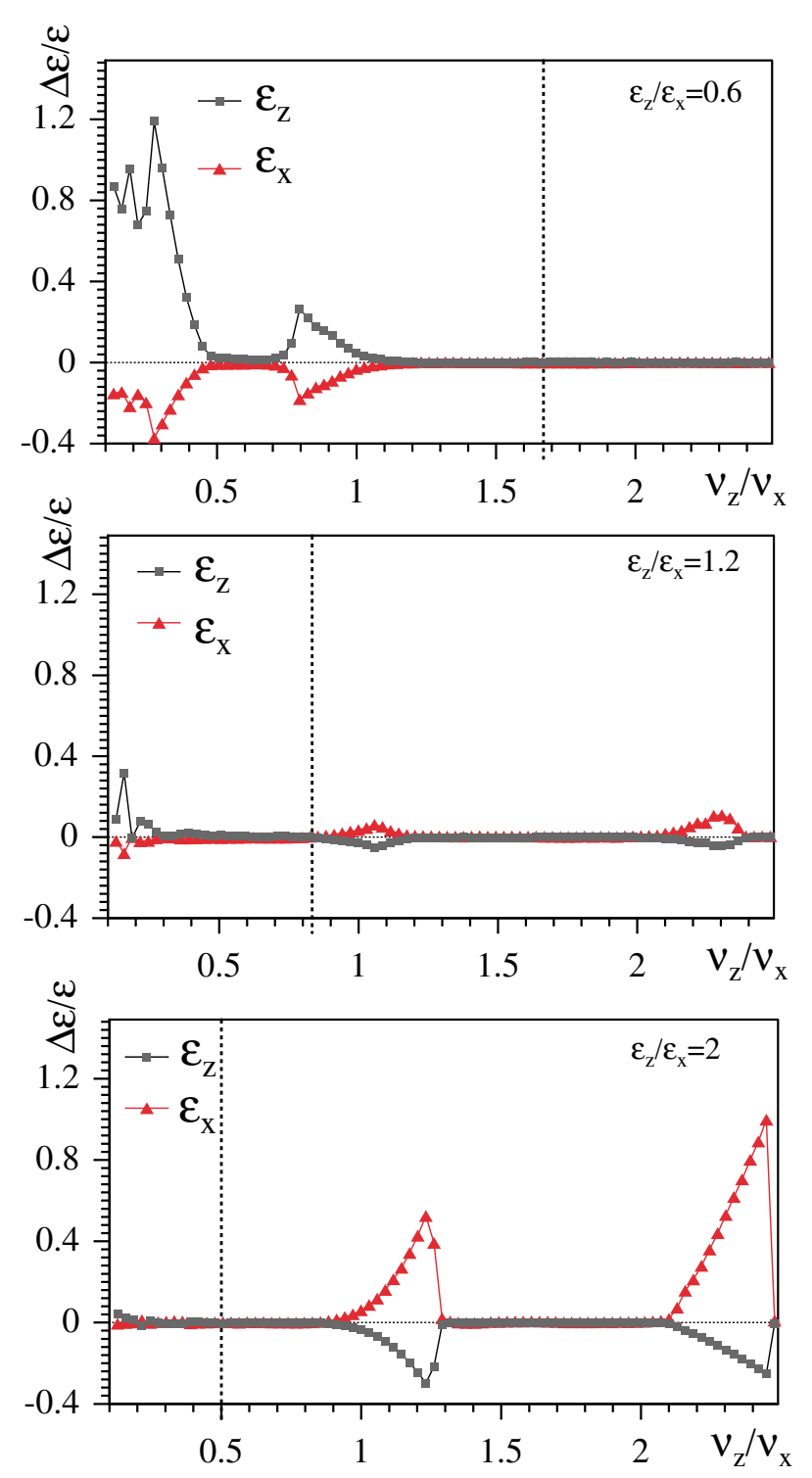

FIG. 9. (Color) Simulation results for WB beams, with $\nu_{x} / \nu_{0 x}=0.5$ and $\epsilon_{z} / \epsilon_{x}=0.6,1.2$, and 2 as functions of the tune ratio (dashed lines equipartitioned).

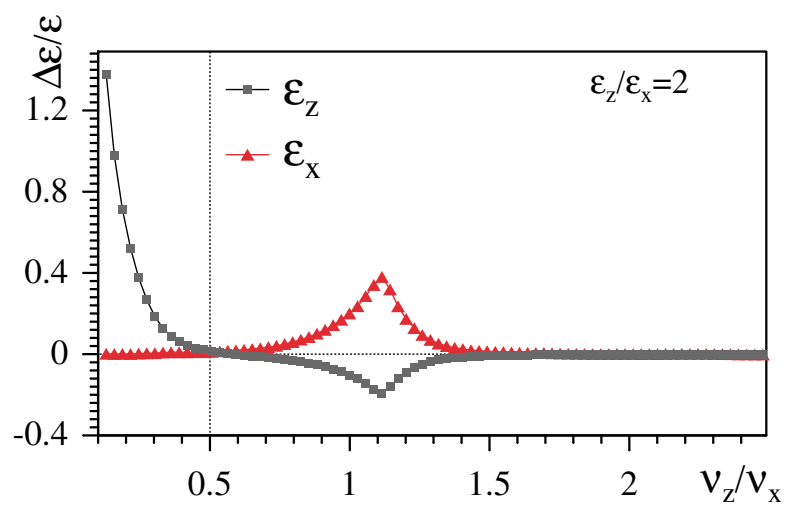

FIG. 10. (Color) Simulation results for $\nu_{x} / \nu_{0 x}=0.5$ and $\epsilon_{z} / \epsilon_{x}=2$ with "frozen-in" space charge force.
The question may be raised if periodic focusing systems would be equally exposed to these resonances. We have examined this question for the example of Fig. 9 $\left(\epsilon_{z} / \epsilon_{x}=2\right)$ and a periodic focusing-drift-defocusingdrift channel and found that the resonant emittance exchange was nearly identical, up to the point where, not surprisingly, the zero current phase advance per period exceeds $90^{\circ}$ in one of the directions and excites the wellknown envelope or fourth order structure resonance [29].

It may be of interest to ask whether the resonant instabilities have an effect beyond the rms emittance exchange. We have examined this for the bottom case of Fig. 9 and found that the ratio of maximum size to rms size increases somewhat in the resonant stop bands, but not exceeding $30 \%$, which is much less than the effect of mismatch induced halos [30]. Hence, equipartitioning is predominantly a core effect not inducing any significant halo.

\section{STABILITY CHARTS}

The above findings are the basis for using the analytical growth rates of nonoscillatory $(\Re \omega=0)$ modes to construct stability charts which indicate the regions of expected resonant exchange for realistic beam models. For practical applications it is convenient to choose the emittance ratio as a given parameter and describe the calculated growth rates in the plane of tune ratio and tune depression in one direction, here chosen as $x$. The evolution of beams along a linac can, in most cases, be characterized on a chart pertinent to the initial emittance ratio, which is hoped to remain invariant. Such charts then indicate the resonant nature of possible emittance exchange. While our findings so far relate only to the coupling between 2 transverse degrees of freedom of a coasting beam, we will show further below, using 3D simulation, that longitudinal-transverse coupling in bunched beams follows similar patterns. In Figs. 11 and 12 we show the overall picture of such "effective stop bands" for different emittance ratios, where the term "effective" expresses the exclusion of the oscillatory KV modes.

Growth rates are understood as maxima over all nonoscillatory modes including second (odd), third, or fourth order. These maxima reach one (zero space charge) betatron period for sufficiently large tune depression. Note that the parameter range of circular accelerators is typically limited to $\nu_{x} / \nu_{0 x}>0.9$. Stop bands in Fig. 11 actually extend to the zero space charge limit at $\nu_{x} / \nu_{0 x}=1$, with growth rates and widths dropping to zero linearly in the space charge tune shift $\Delta \nu_{x} / \nu_{0 x}$, which is not completely resolved by the discrete steps of the contour plots. Note that plotting nonoscillatory unstable modes only discards most of the KV instabilities, except for those, where a negative energy mode turns unstable with $\mathfrak{R} \omega=0$ as may be the case for strong tune depression near the equipartition tune ratio; if we had discarded 

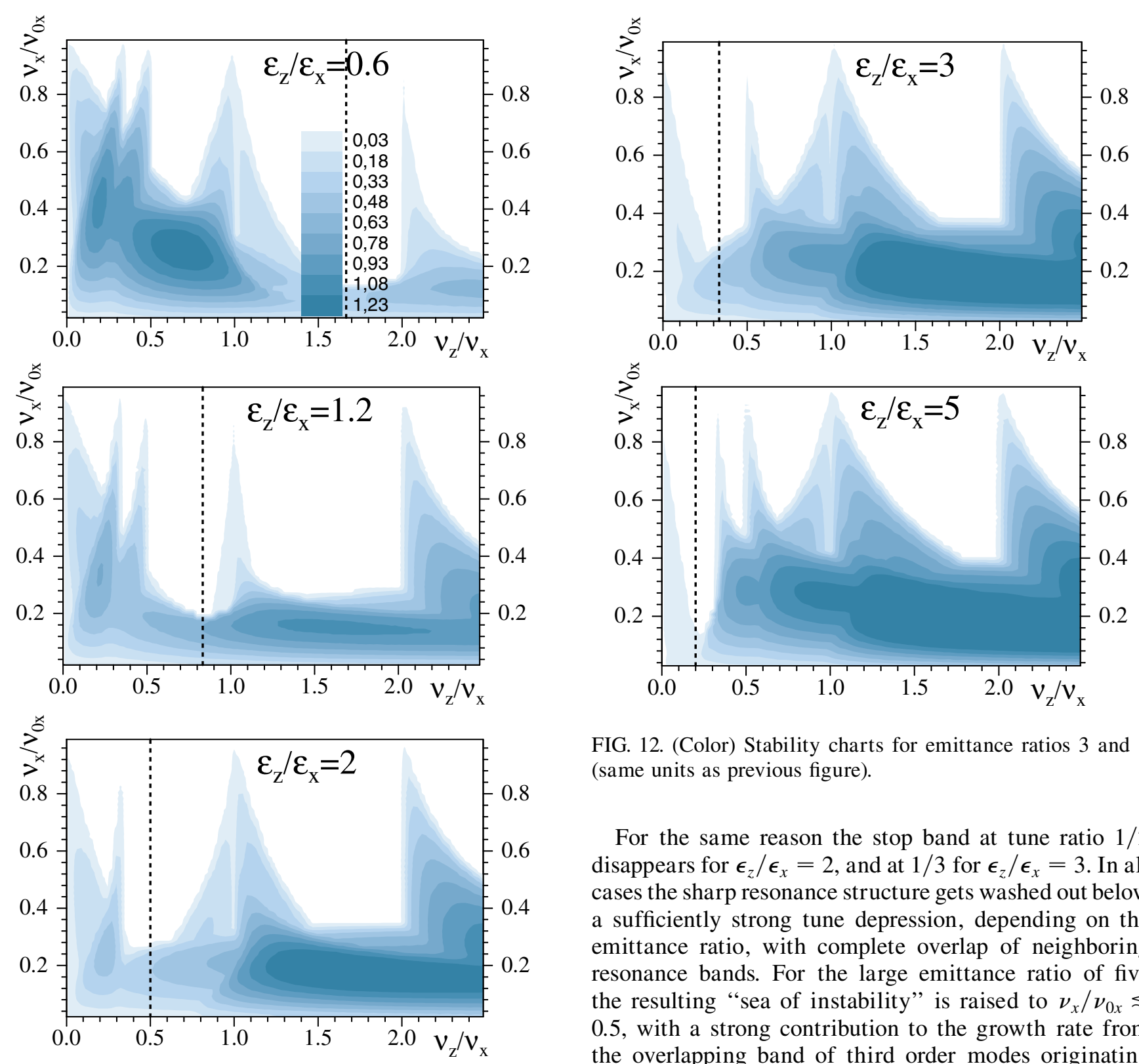

FIG. 12. (Color) Stability charts for emittance ratios 3 and 5 (same units as previous figure).

For the same reason the stop band at tune ratio $1 / 2$ disappears for $\epsilon_{z} / \epsilon_{x}=2$, and at $1 / 3$ for $\epsilon_{z} / \epsilon_{x}=3$. In all cases the sharp resonance structure gets washed out below a sufficiently strong tune depression, depending on the emittance ratio, with complete overlap of neighboring resonance bands. For the large emittance ratio of five the resulting "sea of instability" is raised to $\nu_{x} / \nu_{0 x} \lesssim$ 0.5 , with a strong contribution to the growth rate from the overlapping band of third order modes originating at $\nu_{z} / \nu_{x} \approx 0.5$. In this region emittance/energy exchange in the direction of equipartition is predicted for all tune ratios, but still at varying rates and not necessarily leading to full equipartition. It is noted that growth rates drop to zero when approaching the space charge limit. For $\nu_{x} / \nu_{0 x} \rightarrow 0$ the betatron tunes in both planes vanish, hence coupling rates also approach zero.

these cases from the charts as well, which we found difficult to implement in an automated search of solutions, the equipartition tune ratio would be completely free of instability down to $\nu_{x} / \nu_{0 x}=0$. In high-current linacs the range of interest is typically $0.5<\nu_{x} / \nu_{0 x}<$ 0.8 , where $x$ stands for the direction in which the linac bunch emittance is smaller, usually the transverse.

For completeness, the stop band at tune ratio $\nu_{z} / \nu_{x} \approx 1$ is found to vanish for $\epsilon_{z} / \epsilon_{x}=1$, where the beam is equipartitioned according to Eq. (2).

\section{3D SIMULATION}

To carry out the 3D-2D comparison we use the fully 3D particle-in-cell code IMPACT [31]. Note that we base this comparison on relating the longitudinal $(z)$ direction in $3 \mathrm{D}$ to $z$ in $2 \mathrm{D}$, while both transverse directions in 3Dassuming equal emittances and focusing - are compared with $y$ in 2D. It will be seen that due to the initial energy equipartition between $x$ and $y$ the dynamical evolution of 
the $3 \mathrm{D}$ case is reduced in most cases to effectively an evolution in $r$ and $z$, hence two dimensions only. Naturally, 3D modes breaking the rotational symmetry have no analog in 2D as will be discussed at the end of this chapter.

The simulations were performed on parallel processors using $2 \times 10^{6}$ macroparticles; the space charge calculation was carried out on a $64 \times 64 \times 64$ spatial grid to contain the beam bunch. Computationally, a doubled grid was used, with a modified Green function, to solve the Poisson equation with open boundary conditions. The choice of nonequipartitioned initial distributions in 3D requires some thought. For equipartitioned beams Gluckstern et al. [32] describe a class of exact equilibria as functions of the Hamiltonian. Such an approach, however, does not provide the freedom to generate nonequipartitioned beams. We therefore introduce anisotropy by first defining a spherical WB distribution in $6 \mathrm{D}$ phase space and then adjusting and matching rms moments to obtain the desired emittance and tune ratios (similar to the procedure in $2 \mathrm{D}$ ).

The existence of resonant instabilities in 3D simulations as well is shown in the following examples. We start with an example of parameters, where third order modes are expected. These are not present in the initial distribution, except as noise; hence exponential growth should be visible. We take a focusing system with $\nu_{0 z} / \nu_{0 x}=1.5$ $\left(\epsilon_{z} / \epsilon_{x}=2\right)$ and increase the current such that the resulting working point would be close to the left stop-band edge at $\nu_{z} / \nu_{x}=2$ in Fig. 11. The energy anisotropy $T \equiv$ $\left(\epsilon_{z} \nu_{z}\right) /\left(\epsilon_{x} \nu_{x}\right)$ is as large as 4 at this edge, and we expect the onset of instability. The simulation shows that for $\nu_{z} / \nu_{x}=1.96$ there is negligible emittance change $(<3 \%$ which stems from initial mismatch) over 200 transverse periods. Slightly above the threshold $\left(\nu_{z} / \nu_{x}=\right.$ 2.04) we find a phase of modest energy exchange after about period 50 which saturates at about period 200 and reduces the anisotropy from $T=4.1 \rightarrow 2.6$ (top panel of Fig. 13). The limited energy exchange yet far from equipartition can be explained by self-detuning: the working point drops below $\nu_{z} / \nu_{x}=2$ after initial emittance exchange-a typical resonance phenomenon. For higher currents the exponential growth is accentuated; it takes only a few periods for $\nu_{z} / \nu_{x}=2.94$, where the energy anisotropy drops from 5.9 to about 1.6, hence again without reaching full equipartition (bottom panel of Fig. 13). Comparison with results from a semi-Gaussian initial distribution have shown the same threshold behavior and exponential growth at a comparable rate. Note that Fig. 13, bottom panel, shows a small $(\approx 5 \%)$ growth of the transverse emittance during a fraction of the first period of oscillation. We relate this growth to the nonlinear field energy from initial density redistribution [33].

For a symmetric focusing system with $\nu_{0 z} / \nu_{0 x}=1$ the corresponding trajectory as a function of current is close to the right edge of the stop band adjacent to $\nu_{z} / \nu_{x}=1$ in
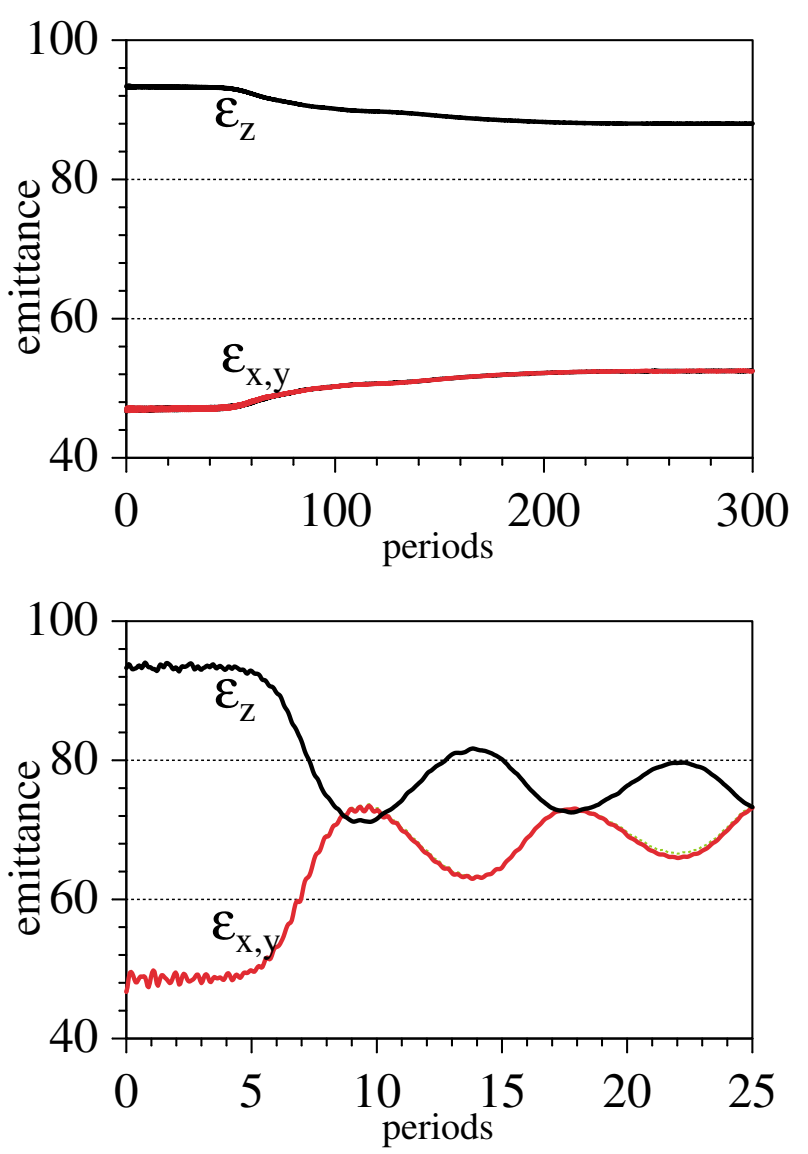

FIG. 13. (Color) rms emittances (arbitrary units) for $\epsilon_{z} / \epsilon_{x}=2$ (WB) and fixed $\nu_{0 z} / \nu_{0 x}=1.5$, both cases inside the resonance band of third order. Top panel: $\nu_{z} / \nu_{x}=2.04(T=4.1 \rightarrow 2.6)$; bottom panel: $\nu_{z} / \nu_{x}=2.94(T=5.9 \rightarrow 1.6)$. Units on the abscissa are transverse single-particle periods without space charge.

Fig. 11, and we expect energy transfer at all current levels, contrary to the system $\nu_{0 z} / \nu_{0 x}=1.5$, which crosses through a resonance band only at a sufficiently high current such that $\nu_{z} / \nu_{x}>2$. This is confirmed by our simulations at various current levels. As an example, we show in Fig. 14 that the exchange takes off immediately, indicating that the instability starts already beyond its early exponential phase as may be expected from the presence of even order modes in the initial density profile mismatch, similar to Fig. 7. The expected $e$-folding of about three periods, according to Fig. 11, is qualitatively in agreement with the simulation. The final emittance as well as the energy ratio saturates at $\approx 1.3$ for all simulated currents of the WB.

In Fig. 15 we summarize results from a large number of simulation cases obtained by performing cuts through parameter space analogous to Figs. 6 and 9.

We retrieve resonant features in emittance exchange, which compare well with those of the 2D simulations and confirm applicability of the stability charts of Fig. 11 to 3D coupling. It is noted that the stop band at $\nu_{z} / \nu_{x} \approx 1$ 


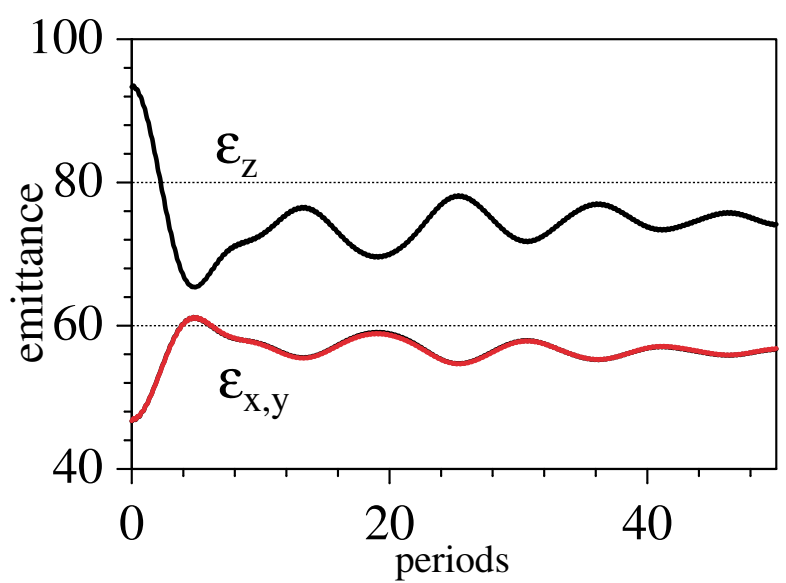

FIG. 14. (Color) Symmetric focusing $\nu_{0 z} / \nu_{0 x}=1, \nu_{z} / \nu_{x}=$ $1.16\left(\nu_{x} / \nu_{0 x}=0.68\right)(\mathrm{WB}:$ with $T: 2.3 \rightarrow 1.3)$.

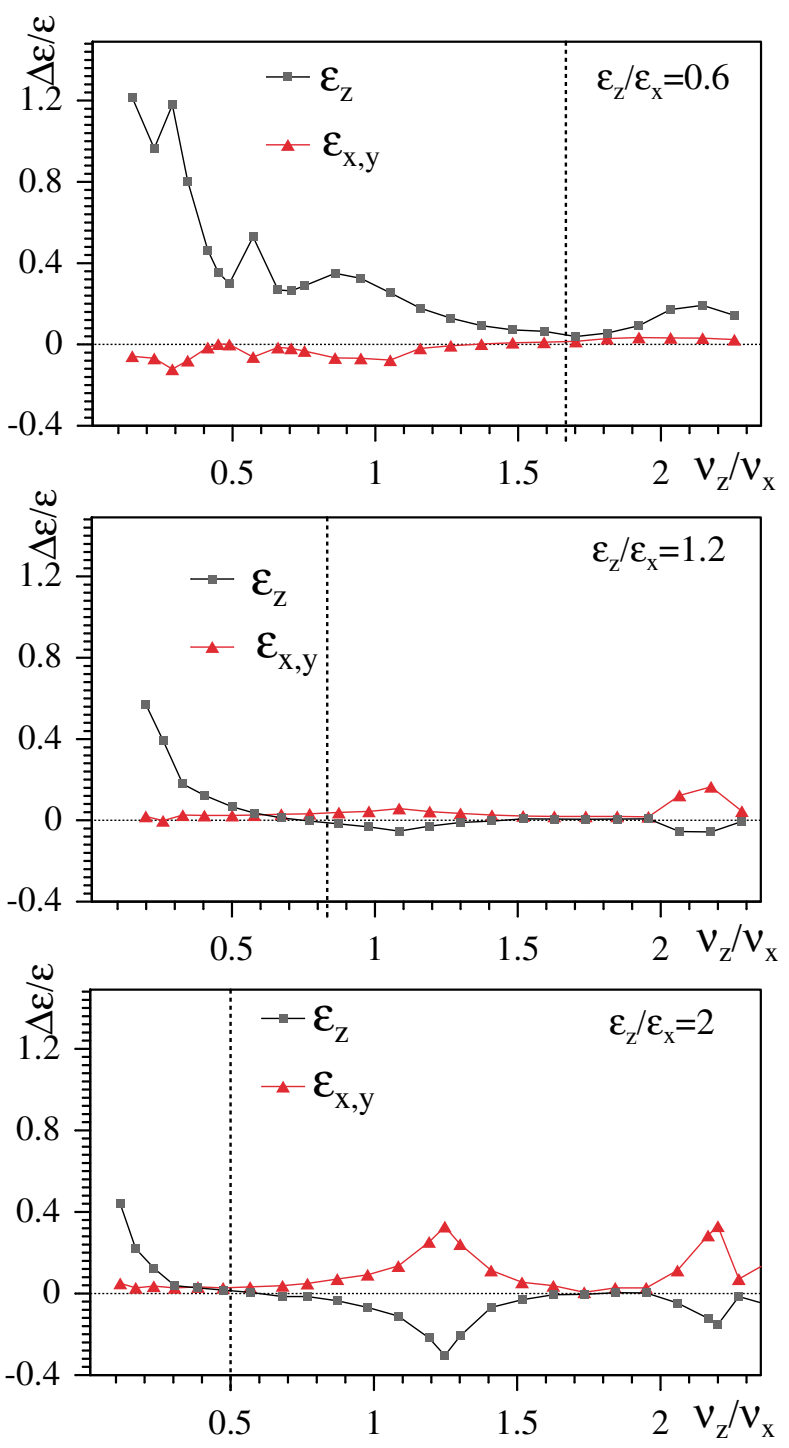

FIG. 15. (Color) 3D IMPACT code simulation results for $\nu_{x} / \nu_{0 x}=0.5$, and $\epsilon_{z} / \epsilon_{x}=0.6,1.2$, and 2 as functions of the tune ratios (dashed lines equipartitioned). obtained for $\epsilon_{z} / \epsilon_{x}=2$ is more symmetric, which suggests that this case is less dominated by collective motion compared with a $2 \mathrm{D} \mathrm{WB}$; the reduced growth in $x$, compared with $2 \mathrm{D}$, is consistent with the fact that the longitudinal excess energy is shared by 2 transverse degrees of freedom.

The pronounced stop band for $\epsilon_{z} / \epsilon_{x}=2$ and $\nu_{z} / \nu_{x} \approx 2$ is again entirely due to collective motion, e.g., the third order coherent difference resonance, similar to $2 \mathrm{D}$. The structure beyond this tune ratio, in particular, the dip at $\nu_{z} / \nu_{x} \approx 2.25$ absent in $2 \mathrm{D}$, may be a fully $3 \mathrm{D}$ phenomenon possibly involving unequal energy exchange between all three directions. There is an indication that the expected initial exchange from longitudinal to both transverse directions may be followed by the exchange of emittances between the 2 (initially equipartitioned) transverse degrees of freedom. While this case occurs in a parameter region beyond linac relevance, it deserves further study from a more general point of view as a pronounced case of $3 \mathrm{D}$ collective resonant motion driving the system away from transverse initial equipartition.

\section{DISCUSSION}

We have shown that the coherent effect of space charge on resonances, described in terms of coherent frequency shifts, may be significant in the parameter regimes relevant to high-intensity linear as well as circular accelerators. Our simulations have shown, moreover, that the gap between analytical theory, based on KV-Vlasov analysis, and realistic 2D and 3D simulations can be largely closed, in particular, by introducing the concept of positive and KV-specific negative energy modes, which can be distinguished according to the coupled mode coefficient. The finding of energy/emittance exchange confined to resonance stop bands, which are relatively narrow for not too strong tune depressions, clarifies that nonequipartitioned beams considered for linac design are a "safe" choice from a beam dynamics point of view, provided that the stop bands are avoided. The proposed stability charts make it possible to correlate design trajectories in tune space with the resonant regions, where emittance transfer/ growth is predicted. From a theoretical point of view it may be worth to extend these studies to beams very close to the space charge limit $\left(\nu_{x} / \nu_{0 x} \leq 0.2\right)$, where approach to equipartition may be a universal feature due to complete overlap of the resonance bands.

\section{ACKNOWLEDGMENTS}

The authors acknowledge valuable comments by R. A. Jameson and M. Reiser. This work was performed using resources of the NERSC and ACL scientific computing centers located at LBNL and LANL, respectively, as well as the CDIC at BNL with support by A. Luccio. The 
hospitality and support of the SNS Project Office and of the AGS Division at Brookhaven National Laboratories during part of the work are acknowledged by two of the authors (I. H. and G. F.).

[1] S. Machida and M. Ikegami, in Proceedings of the Workshop on Space Charge Physics in High Intensity Hadron Rings, AIP Conf. Proc. No. 448 (AIP, New York, 1998), p. 73.

[2] M. Venturini and R. L. Gluckstern, Phys. Rev. ST Accel. Beams 3, 034203 (2000).

[3] R. Baartman, in Proceedings of the Workshop on Space Charge Physics in High Intensity Hadron Rings (Ref. [1]), p. 56.

[4] A.V. Fedotov and I. Hofmann, Phys. Rev. ST Accel. Beams 5, 024202 (2002).

[5] S. Strasburg and R. C. Davidson, Phys. Rev. E 61, 5753 (2000).

[6] R. A. Jameson, in Proceedings of the Third Workshop on Advanced Accelerator Concepts, Port Jefferson, 1992, AIP Conf. Proc. No. 279 (AIP, New York, 1992), p. 969.

[7] I. Hofmann, Phys. Rev. E 57, 4713 (1998).

[8] M. Reiser and N. Brown, Phys. Rev. Lett. 74, 1111 (1995).

[9] M. Reiser, Theory and Design of Charged Particle Beams (Wiley-Interscience, New York, 1994).

[10] R. A. Kishek, P. G. O'Shea, and M. Reiser, Phys. Rev. Lett. 85, 4514 (2000).

[11] I. Hofmann, J. Qiang, and R. Ryne, Phys. Rev. Lett. 86, 2313 (2001).

[12] B.W. Montague, CERN Report No. 68-38, 1968.

[13] I. Hofmann and O. Boine-Frankenheim, Phys. Rev. Lett. 87, 034802 (2001).

[14] I. Hofmann, G. Franchetti, O. Boine-Frankenheim, J. Qiang, R. Ryne, D. Jeon, and J. Wei, in Proceedings of the Particle Accelerator Conference, Chicago, 2001, edited by Y. Cho (IEEE, Piscataway, NJ, 2001), p. 2902.
[15] F. Gerigk and I. Hofmann, in Proceedings of the Particle Accelerator Conference, Chicago, 2001 (Ref. [14]), p. 2872.

[16] I. Hofmann, G. Franchetti, J. Qiang, R. Ryne, F. Gerigk, D. Jeon, and N. Pichoff, in Proceedings of the European Accelerator Conference, Paris, 2002, edited by J. L. Laclare (CERN, Geneva, 2002), p. 74 (http://accelconf.web.cern.ch/AccelConf/e02/default.htm).

[17] T.-W. Wang and L. Smith, Part. Accel. 12, 247 (1982).

[18] E. A. Startsev, R.C. Davidson, and Q. Qian, Phys. Plasmas 9, 3138 (2002).

[19] E. G. Harris, Phys. Rev. Lett. 2, 34 (1959).

[20] F. Sacherer, Ph.D. thesis, University of California [Lawrence Radiation Laboratory Report No. UCRL18454, 1968 (unpublished)].

[21] I. Hofmann and K. Beckert, IEEE Trans. Nucl. Sci. 32, 2264 (1985).

[22] M. Conte and W.W. MacKay, An Introduction to the Physics of Particle Accelerators (World Scientific, Singapore, 1991).

[23] R. L. Gluckstern, in Proceedings of the Linac Conference, 1970 (Fermilab, Batavia, IL, 1970), p. 811.

[24] I. Hofmann, Part. Accel. 10, 253 (1980).

[25] I. Hofmann, Phys. Fluids 23, 296 (1980).

[26] S. M. Lund and R. C. Davidson, Phys. Plasmas 5, 3028 (1998).

[27] A. V. Fedotov, J. Holmes, and R. L. Gluckstern, Phys. Rev. ST Accel. Beams 4, 084202 (2001).

[28] G. Franchetti, I. Hofmann, and G. Turchetti, in Proceedings of the Workshop on Space Charge Physics in High Intensity Hadron Rings (Ref. [1]), p. 233.

[29] I. Hofmann, L. J. Laslett, L. Smith, and I. Haber, Part. Accel. 13, 145 (1983).

[30] T. P. Wangler, Phys. Rev. ST Accel. Beams 1, 084201 (2000).

[31] J. Qiang et al., J. Comput. Phys. 163, 434 (2000).

[32] R. L. Gluckstern et al., Phys. Rev. E 58, 4977 (1998).

[33] I. Hofmann and J. Struckmeier, Part. Accel. 21, 69 (1987). 